\title{
Kinematics of the disk working body for ground development
}

\author{
Ramil Gainutdinov $^{1 *}$, and Minsur Zemdikhanov ${ }^{1[0000-0001-5207-2346]}$ \\ ${ }^{1}$ Kazan State University of Architecture and Engineering, 420043 Kazan, Russia
}

\begin{abstract}
High rates of growth in the volume of earthworks in winter, with a lack of funds for the purchase of new equipment, determine the relevance of work to improve the existing and create new working bodies for the development of frozen grounds and snow-ice formations to increase the efficiency of their destruction without increasing the power of the base machine. This can be done by installing a fundamentally new cutting tool in the form of elliptical circular knives on the working bodies. The article deals with the kinematics of an ellipsoidal disk working body. Expressions for determining the displacement, velocity and acceleration of the point of the cutting edge of the disk are obtained analytically. It was found that the points of the cutting edge of the disk move in space along a complex trajectory, and the absolute values of velocity and acceleration are variable parameters. The regularity of the change in the angle of entry of the disk into the ground at different values of the angle of its attachment to the shaft of the working battery was revealed, and the main design and technological parameters were determined and substantiated.
\end{abstract}

Keywords. Ellipsoidal disc, circular knive, kinematics, frozen ground.

\section{Introduction}

Further development of the north and the territories adjacent to the Baikal-Amur Mainline will entail an increase in the development of frozen grounds [1-18]. The construction of oil and gas fields in Siberia requires the construction of transport routes, cable networks, drilling of wells and loosening of frozen ground $[9,19]$. Due to the swampy terrain, the construction of pipelines to the Far East and to China is a particularly urgent problem of mechanically accelerating the development of frozen grounds and snow-ice formations in winter $[1,17,18]$.

High rates of growth in the volume of earthworks in winter with a lack of funds for the purchase of new equipment determine the relevance of work to improve the existing and create new working bodies for the development of frozen grounds and snow-ice formations to increase the efficiency of their destruction without increasing the power of the base machine $[2,12,13]$. This can be done by installing a fundamentally new cutting tool in the form of elliptical circular knives on the working bodies.

An integral part of the design stage of machines is to ensure the strength of structural elements, reduce energy costs, as well as identify the conditions for the stability of the movement of the unit $[5,6,14,15]$.

*Corresponding author: grh1978@mail.ru 
The developed working body for ground processing contains elliptical disks, fixed on the shaft of the working battery strictly at a certain angle [20]. It is impossible to analyze the process of interaction of the proposed working body with the ground and substantiate the main parameters of the technology without studying the kinematics of the ellipsoidal disk [4, 7].

The purpose of this paper is to study the kinematics of the disk operating element, namely the construction of movement trajectories, velocity and acceleration of point cutting edge of the disk.

\section{Methods}

When studying the kinematics of a flat circular disk fixed on a shaft at a certain angle, some authors $[8,11]$ make the assumption that the distance of the point of the cutting edge of the disk from the axis of rotation is a constant value, and the absolute velocity vector is equal to the sum of the vectors of the translational velocity of the disk and the velocity of the disk in harmonic vibration mode. This technique, as calculations show, distorts the real picture $[10,16]$.

To obtain the main kinematic dependences, in contrast to the above technique, we considered the motion of a point $M$ arbitrarily selected on the cutting edge of an ellipsoidal disk (hereinafter referred to as a disk) in a spatial rectangular coordinate system (Fig. 1).

To conduct laboratory research in accordance with the developed design and technical documentation and in accordance with the developed program, an experimental installation was made in the laboratory of the departmen (Fig. 2).

Research methodology and methods. Theoretical studies used the basics of mathematical analysis, analytical geometry, theoretical mechanics and basic methods of agricultural mechanics.

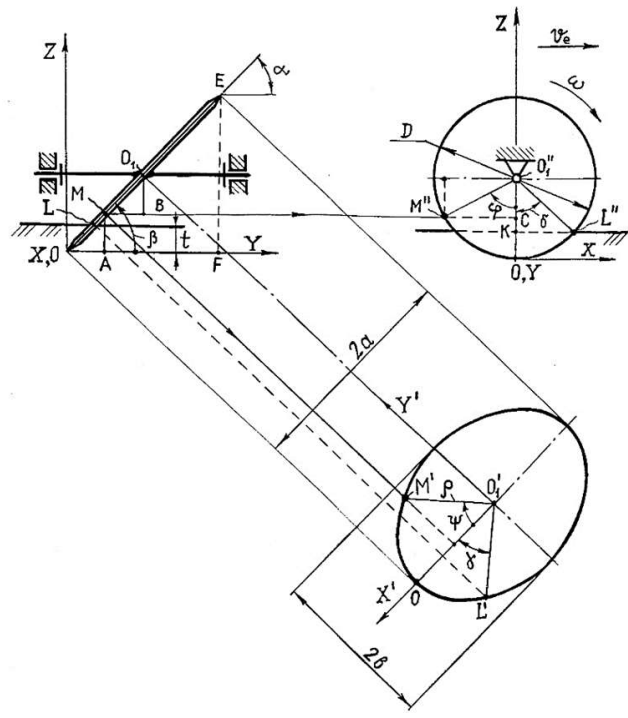

Fig. 1. Elliptical disk.

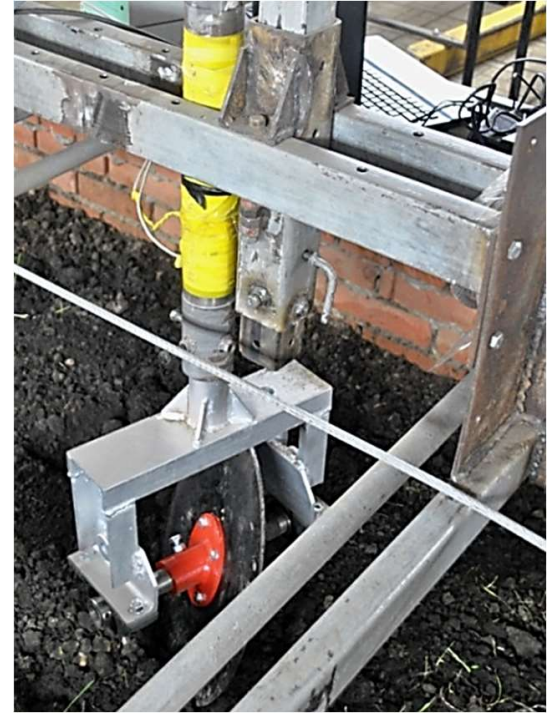

Fig. 2. Laboratory setup.

The diameter of the disk $D$ in the profile plane (hereinafter - the diameter of the disk), the angle of inclination $\alpha$ of the major axis of the ellipse to the axis of rotation of the disk, and the translational speed of the machine $V_{e}$ are given. The position of the investigated point in the profile plane is characterized by the polar angle $\varphi=\omega t$ (hereinafter referred to as the angle of rotation of the disk). Disks are «passive», so the angular velocity of their rotation is calculated according to the well-known formula: $\omega=2 V_{e} / D$.

First, we determine the abscissa of point $M$ :

$$
X_{M}=V_{e} t-M " C=V_{e} t-D \sin \varphi / 2 .
$$


Substituting in expression (1) the known values $V_{e}=\omega D / 2, t=\varphi / \omega$, we have:

$$
X_{M}=D(\varphi-\sin \varphi) / 2 \text {. }
$$

Now you can define the fingering point for the point of interest. As can be seen from Fig. 4, it is equal to the length of the segment $A M$ :

$$
Z_{M}=A M=\frac{D}{2}-O_{1}^{\prime \prime} C=\frac{D}{2}-\frac{D}{2} \cos \varphi=D(1-\cos \varphi) / 2
$$

To determine the ordinate of point $M$, consider the right-angled triangle OMA:

$$
Y_{M}=O A=A M / \operatorname{tg} \alpha=Z_{M} / \operatorname{tg} \alpha=D(1-\cos \varphi) /(2 \operatorname{tg} \alpha) \text {. }
$$

Thus, the parametric equations of motion of the point $\mathrm{M}$ of the cutting edge of the disk in the spatial rectangular coordinate system $\mathrm{OXYZ}$ were analytically obtained:

$$
\left.\begin{array}{c}
X_{M}=D(\varphi-\sin \varphi) / 2, \\
Y_{M}=D(1-\cos \varphi) /(2 \operatorname{tg} \alpha), \\
Z_{M}=D(1-\cos \varphi) / 2
\end{array}\right\} .
$$

The magnitude (modulus) of movement $S$ of point $M$ is determined by the formula:

$$
S=\sqrt{X_{M}^{2}+Y_{M}^{2}+Z_{M}^{2}}=\frac{D}{2} \sqrt{(\varphi-\sin \varphi)^{2}+[(1-\cos \varphi) / \sin \alpha]^{2}} .
$$

Differentiating expression (5) with respect to time, it is possible to calculate the projection of the velocity of the point under study. So:

$$
\left.\begin{array}{c}
V_{M}^{X}=d X_{M} / d t=D \omega(1-\cos \varphi) / 2, \\
V_{M}^{Y}=d Y_{M} / d t=D \omega \sin \varphi /(2 \operatorname{tg} \alpha) \\
V_{M}^{Z}=d Z_{M} / d t=D \omega \sin \varphi / 2 .
\end{array}\right\} .
$$

The magnitude (modulus) of the absolute speed is determined from the formula:

$$
V_{M}=\sqrt{\left(V_{M}^{X}\right)^{2}+\left(V_{M}^{Y}\right)^{2}+\left(V_{M}^{Z}\right)^{2}}=\frac{D \omega}{2} \sqrt{2(1-\cos \varphi)+(\sin \varphi / \operatorname{tg} \alpha)^{2}} .
$$

Differentiating expression (7) with respect to time gives the projection of the acceleration of point $M$ :

$$
\left.\begin{array}{c}
a_{M}^{X}=d V_{M}^{X} / d t=D \omega^{2} \sin \varphi / 2, \\
a_{M}^{Y}=d V_{M}^{Y} / d t=D \omega^{2} \cos \varphi /(2 \operatorname{tg} \alpha), \\
a_{M}^{Z}=d V_{M}^{Z} / d t=D \omega^{2} \cos \varphi / 2 .
\end{array}\right\} .
$$

The magnitude (modulus) of the absolute acceleration is determined by the formula:

$$
a_{M}=\frac{D \omega^{2}}{2} \sqrt{1+(\cos \varphi / \operatorname{tg} \alpha)^{2}} .
$$

The direction of the vectors of velocity and acceleration of the investigated point $M$ of the cutting edge of the disk in space is determined by the so-called direction cosines, which are calculated, respectively, by the formulas:

$$
\left.\begin{array}{c}
K_{X}^{V_{M}}=V_{M}^{X} / V_{M}=(1-\cos \varphi) / \sqrt{2(1-\cos \varphi)+(\sin \varphi / \operatorname{tg} \alpha)^{2}}, \\
K_{Y}^{V_{M}}=V_{M}^{Y} / V_{M}=\sin \varphi /\left(\operatorname{tg} \alpha \sqrt{2(1-\cos \varphi)+(\sin \varphi / \operatorname{tg} \alpha)^{2}}\right), \\
K_{Z}^{V_{M}}=V_{M}^{Z} / V_{M}=\sin \varphi / \sqrt{2(1-\cos \varphi)+(\sin \varphi / \operatorname{tg} \alpha)^{2}} . \\
K_{X}^{a_{M}}=a_{M}^{X} / a_{M}=\sin \varphi / \sqrt{1+(\cos \varphi / \operatorname{tg} \alpha)^{2}}, \\
K_{Y}^{a_{M}}=a_{M}^{Y} / a_{M}=\cos \varphi /\left(\operatorname{tg} \alpha \sqrt{1+(\cos \varphi / \operatorname{tg} \alpha)^{2}}\right), \\
K_{Z}^{a_{M}}=a_{M}^{Z} / a_{M}=\cos \varphi / \sqrt{1+(\cos \varphi / \operatorname{tg} \alpha)^{2}} .
\end{array}\right\} .
$$

Now we determine the value of the polar radius of the disk, that is, the radius of the ellipse in the plane of the cutting edge $\rho=O_{I}{ }^{\prime} M$. To do this, we will use the canonical equation of the ellipse, which has the form:

$$
\left(X^{\prime} / a\right)^{2}+\left(Y^{\prime} / b\right)^{2}=1 .
$$

Substituting into equation (13) the values of the semiaxes $a=D /(2 \sin \alpha), b=D / 2$ and the current coordinates of the point $M^{\prime}$ in a Cartesian coordinate system $O_{1}^{\prime} X^{\prime} Y^{\prime}: X^{\prime}=\rho \cos \psi$, $Y^{\prime}=\rho \sin \psi$, after some transformations, we get an expression for calculating the polar radius of the disk. It looks like:

$$
\rho=D /\left(2 \sqrt{\sin ^{2} \psi+(\sin \alpha \cos \psi)^{2}}\right) .
$$


Kinematic parameters for a given condition are determined depending on the angle of rotation of the disk $\varphi$. When justifying the design parameters, we are faced, however, with the angle $\psi$, which characterizes the position of the considered point $M$ in the plane of the cutting edge. To determine the mathematical relationship between the angles $\varphi$ and $\psi$, we express the application of the point $M$ through the angle $\psi$ :

$$
Z_{M}=D / 2-O_{l} \mathrm{~B} \text {. }
$$

Since $O_{1} B=M O_{1} \sin \alpha$, and in turn, $M O_{1}=\rho \cos \psi$, formula (15) takes the following form:

$$
Z_{M}=\frac{D}{2}-\rho \cos \psi \sin \alpha
$$

Based on expressions (3) and (16), we can write:

$$
\frac{D(1-\cos \varphi)}{2}=\frac{D}{2}-\rho \cos \psi \sin \alpha \text {. }
$$

Substituting into equation (17) the value of $\rho$ from formula (14), after certain transformations and simplifications, we finally have:

$$
\psi=\arcsin \left(\sin \alpha \sin \varphi / \sqrt{\cos ^{2} \varphi+(\sin \alpha \sin \varphi)^{2}}\right) .
$$

As expression (18) shows, the angle $\psi$ is generally not proportional to the angle $\varphi$. However, for angles equal to $0^{\circ}, 90^{\circ}, 270^{\circ}$ and $360^{\circ}, \psi=\varphi$, which can be clearly seen from Fig. 1 .

During the technological process, the disks enter the ground at different angles $\beta$. Let us reveal the regularity of this angle change, since it is necessary in the future when determining individual technological parameters. In the general case, from the right-angled triangle OEF we have:

$$
\sin \beta=D /(2 \rho) \text {. }
$$

Substituting into expression (19) the value of the polar radius $\rho$ from dependence (14), we obtain a formula for determining the angle $\beta$, which has the form:

$$
\beta=\arcsin \sqrt{\sin ^{2} \psi+(\sin \alpha \cos \psi)^{2}} \text {. }
$$

Only a certain part of the cutting edge of the disk is involved in the process of work. The length of the edge section cutting the seam is equal to the arc length $O L^{\prime}$ and is calculated using the following equation [3]:

$$
d l_{p}=\sqrt{\left(d X_{L^{\prime}}\right)^{2}+\left(d Y_{L^{\prime}}\right)^{2}+\left(d Z_{L^{\prime}}\right)^{2}}
$$

where $X_{L^{\prime}}, Y_{L^{\prime}}, Z_{L^{\prime}}$ - coordinates of point $L$.

Due to the fact that the length of the arc $O L^{\prime}$ in this case is part of the perimeter of the ellipse, equation (21) is reduced to the form:

$$
l_{p}=a \int_{0}^{\gamma} \sqrt{1-\varepsilon^{2} \sin ^{2} \gamma} d \gamma,
$$

where $a$ is the semi-major axis of the ellipse, $\varepsilon$ is the eccentricity of the ellipse.

As known,

$$
\varepsilon=\sqrt{a^{2}-b^{2}} / a
$$

After substituting the values of the semiaxes $a$ and $b$ into this formula, we obtain that $\varepsilon=\cos \alpha$.

To determine the upper limit of integration in expression (22), i.e., the angle $\gamma$, we first determine the cosine of the angle $\delta$ from a right-angled triangle $K O_{1}^{\prime \prime} L^{\prime \prime}$ :

$$
\cos \delta=O_{1}^{\prime \prime} K / O_{1}^{\prime \prime} L^{\prime \prime} \text {. }
$$

Therefore, after substituting the values of the corresponding segments into formula (24), we have: where $t$ is the processing depth.

$$
\delta=\arccos [(1-2 t) / D] \text {. }
$$

Then, using formula (18), we pass to the sought angle $\gamma$ :

$$
\gamma=\arcsin \left(\sin \alpha \sin \delta / \sqrt{\cos ^{2} \delta+(\sin \alpha \sin \delta)^{2}}\right)
$$

During one revolution, the investigated point $M$ of the cutting edge of the disk travels a path equal to the perimeter of the ellipse:

$$
L_{B}=4 a \int_{0}^{\gamma=\pi / 2} \sqrt{1-\cos ^{2} \alpha \sin ^{2} \gamma} d \gamma
$$


The elliptic integrals of expressions (22) and (27) cannot be calculated in the usual way. To solve them, ready-made tabular data are used [3].

\section{Results and discussion}

As a result of calculations, the corresponding graphs were built.

The point of the cutting edge of the disk makes a complex spatial movement during movement (Fig. 3a). In the horizontal and vertical planes, it moves along a sinusoidal trajectory (Fig. 3b), which contributes to effective crumbling of the ground.

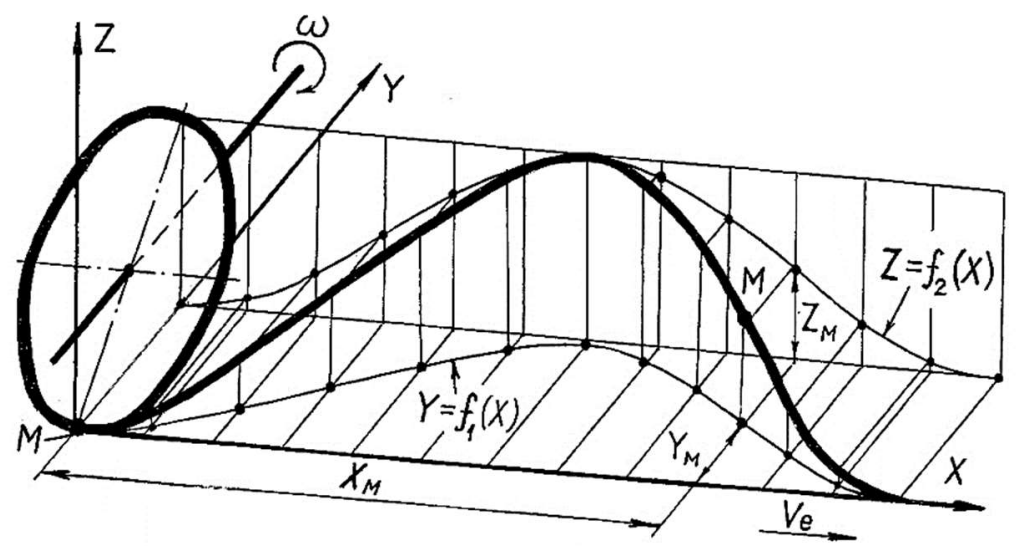

a)

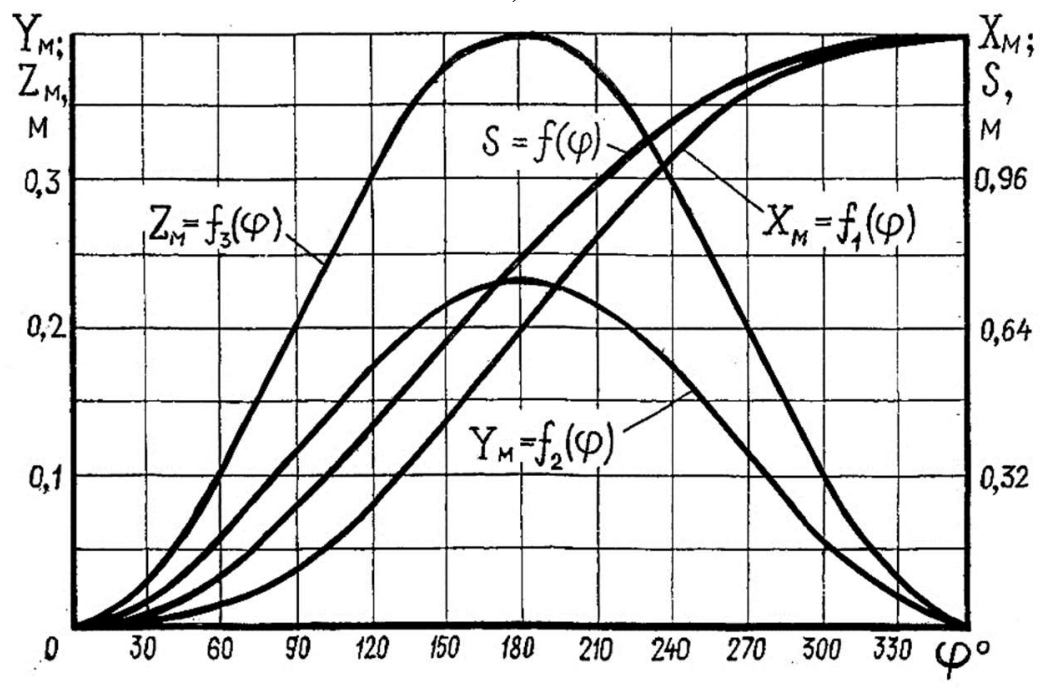

b)

Fig. 3. Trajectory (a) and displacement graphs (b) of the point of the cutting edge of an elliptical disk.

As for the components of the speed of the point under study (Fig. 4), the nature of their changes in different planes is different. Velocity components directed along the $Y$ and $Z$ axes twice in one revolution at angles of rotation $\varphi$ equal to $90^{\circ}$ and $270^{\circ}$ reach their extreme values, and at $\varphi=0^{\circ}$ and $\varphi=180^{\circ}$ they are equal to zero. The velocity component $V_{M}^{X}$, on the contrary, reaches its maximum value at $\varphi=180^{\circ}$, and at $\varphi=0^{\circ}$, the component $V_{M}^{X}=0$. 


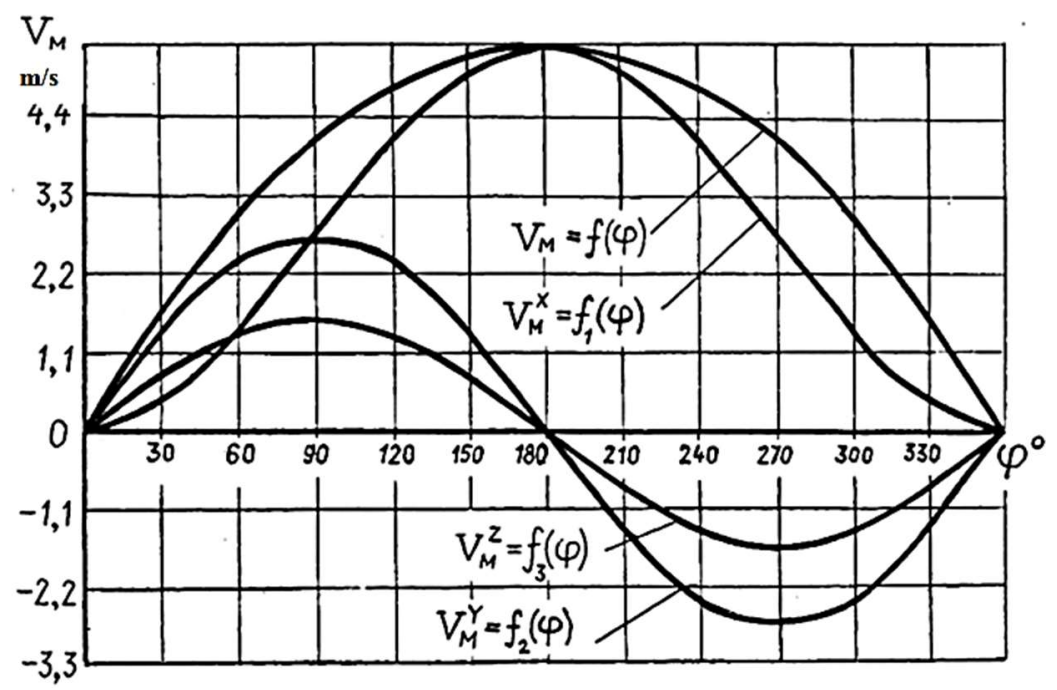

Fig. 4. Graphs of speeds of a point of a cutting edge of a disk.

The projections of the acceleration of point $\mathrm{M}$ of the cutting edge of the disk, as shown by the graphs (Fig. 5), change both their values and directions depending on the angle $\varphi$.

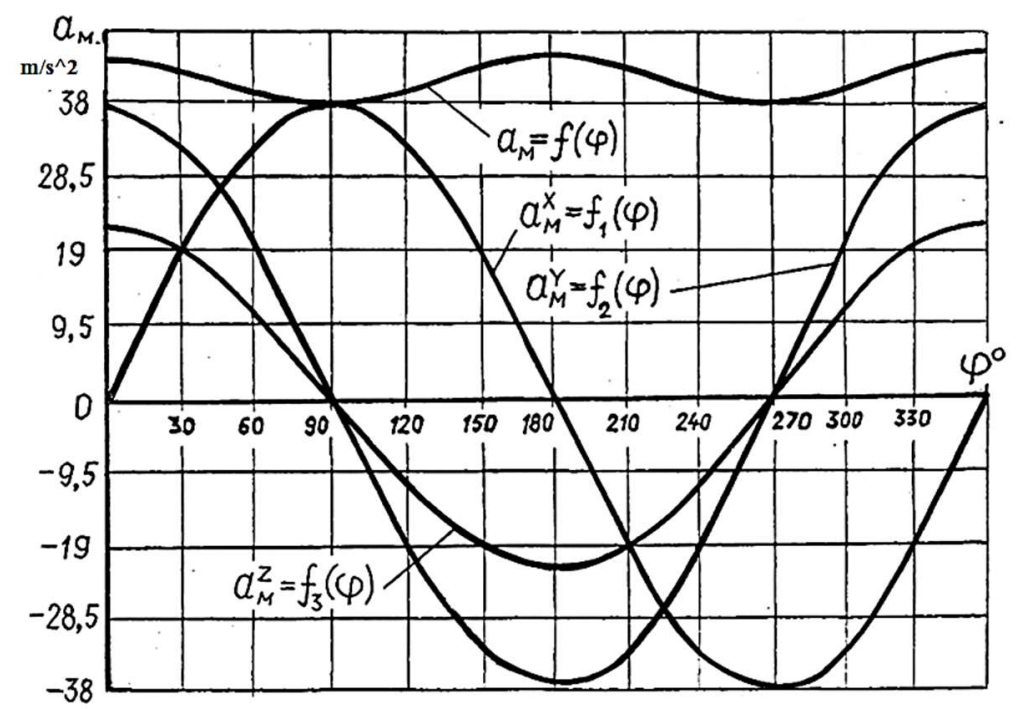

Fig. 5. Graphs of accelerations of the point of the cutting edge of the disk.

Acceleration components $a_{M}^{Z}$ and $a_{M}^{Y}$ at $\varphi=0^{\circ}$ and $\varphi=180^{\circ}$, i.e. twice in one revolution, reach their extreme values, and at angles $\varphi$ equal to $90^{\circ}$ and $270^{\circ}$, these components of acceleration are equal to zero. It is also seen that the acceleration $a_{M}^{X}$ per revolution twice at angles $\varphi$ equal to $90^{\circ}$ and $270^{\circ}$ reaches its maximum value, and at $\varphi=0^{\circ}$ and $\varphi=180^{\circ}, a_{M}^{X}=0$.

The variable nature of the components of the velocity and acceleration of the points of the cutting edge contributes to the intensive self-cleaning of the disks from the adhered ground. With an increase in the forward speed of the unit, this effect increases.

From the graphs presented in Fig. 6, it can be seen that the angle of entry of the disk into the ground is a variable value. 


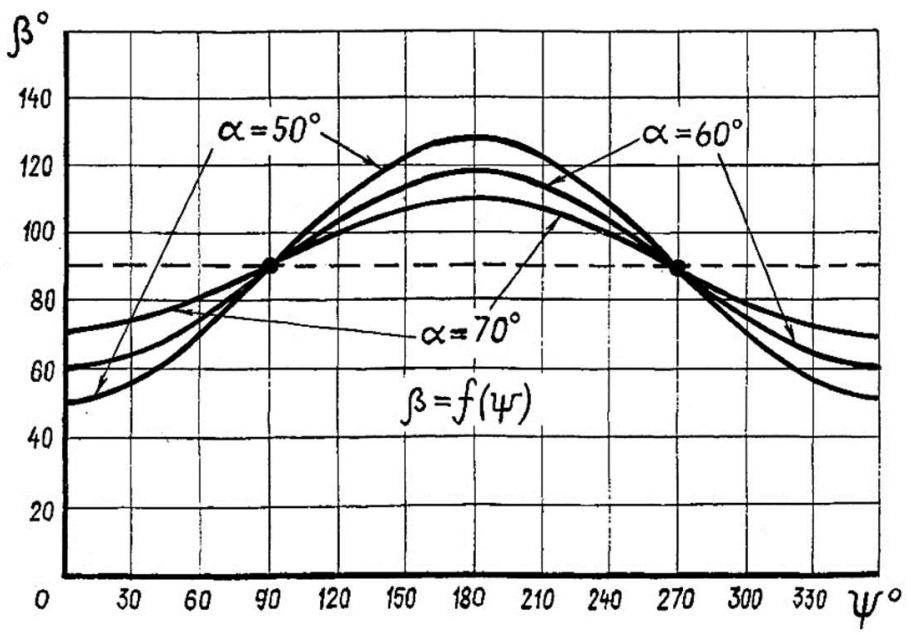

Fig. 6. Graphs of changing the angle of entry of disks into the ground.

The approach angle reaches its minimum value at $\psi=0^{\circ}$, while $\beta_{\min }=\alpha$, and the maximum value - at $\psi=180^{\circ}$, then $\beta_{\max }=\left(180^{\circ}-\alpha\right)$. It is also seen that the disk enters the ground at a right angle twice during one revolution at angles $\psi$ equal to $90^{\circ}$ and $270^{\circ}$. Here we note that in the design scheme, due to the specifics of the design, at angles $\psi$ equal to from $90^{\circ}$ to $270^{\circ}$, the counterclockwise reference system of angle $\beta$ is violated. Therefore, in the specified interval, the values of this angle are determined from the following expression: $\beta^{\prime}=\left(180^{\circ}-\beta\right)$, where $\beta$ is the value of the angle of approach of the disk, calculated by the formula (20).

\section{Conclusions}

Laboratory studies have shown that the constancy of the distance from the cutting edge of the disks to the axis of rotation significantly improves the dynamics of the movement of the machine, and thus the technological stability. The movement becomes stable, that is, there is no vertical vibration. The disks smoothly enter the ground, the conditions for sliding cutting of the formation are stabilized, and the uniformity of the working depth of processing is ensured.

Having analyzed the parametric equations of motion obtained by the method of analytical geometry and the corresponding graphs built on their basis, we can draw the following conclusions:

1) points of the cutting edge of the disk move in space along a complex trajectory, in particular in the horizontal and vertical planes, they move along a sinusoidal trajectory;

2 ) the components of the velocity and acceleration of the point are variable parameters, which contributes to the active destruction of the ground and self-cleaning of the disks from various residues.

\section{References}

1. V.I. Balovnev. Road-building machines with working bodies of intensifying action. M.: Mashinostroenie, 223 (1981).

2. L.I. Baron. Destruction of rocks by roadheaders. Destruction by tangential tool. M.: Nauka, 172 (1973).

3. A.F. Bermant, I.G. Aramanovich. Short course of mathematical analysis. St. Petersburg: Lan, 735 (2005). 
4. R.Kh. Gainutdinov. Substantiation of the parameters of a rotary tool with elliptical disks, Bulletin of Kazan State Agrarian University 1 (43), 71-75 (2017).

5. GOST 29194-91 Earth-moving machines. Tractors. Terminology and technical 142 characteristics for commercial documentation. M.: IPK Standards Publishing House, (2003).

6. GOST R ISO 6165-99 Earth-moving machines Classification. Terms and Definitions. (Earth - moving machinery. Basic types. Vocabulary). M.: IPK Standards Publishing House, 2000.

7. V.N. Kuznetsova, A.M. Zavyalov. Development of frozen soils by earth-moving machines. Omsk: Orange, 224 (2011).

8. M.M. Makhmutov, R.M. Mukhametshina, M.M. Zemdikhanov, A.M. Zaripov. Reliability of machines in road construction, Izvestiya KGASU 4 (46), 358-357 (2018).

9. A.F. Nuriev, R.A. Ibragimov. Improving the efficiency of construction of buildings and structures in extreme climatic conditions through the use of energy-saving technologies, Izvestiya KGASU 2 (48), 272-278 (2019).

10. F.B. Piotr, Marek Klimkiewicz, Małgorzata Powałka. Selected problems of soil tillage systems and operations. Warsaw: Warsaw University of Life Sciences, 133 (2010).

11. I.K. Rastegaev. Machines for permafrost grounds. M.: Mashinostroenie, 216 (1986).

12. R.L. Sakhapov, N.K. Mazitov, R.S. Rakhimov, Ya.P. Lobachevsky, N.Kh. Galyautdinov, L.Z. Sharafiev. Equipment for the village 3 (189), 2-6 (2013).

13. K.K. Shestopalov. Construction and road machines. M.: ITs Academy, 384 (2008).

14. R. Sakhapov, M. Makhmutov. Optimizing the number of anti-skid device for vehicle, IOP Conference Series: Materials Science and Engineering 786, 012038 (2020). DOI: 10.1088/1757-899X/786/1/012038.

15. R. Sakhapov, M. Makhmutov. Influence of removable anti-skid device on the soil density, IOP Conference Series: Materials Science and Engineering 786, 012021 (2020). DOI: 10.1088/1757-899X/786/1/012021.

16. A.S. Soyunov Substantiation of the parameters of disk working bodies of tillage tools. Omsk, 19 (2011).

17. Ajit K. Srivastava, Carroll E. Goering, Roger P. Rohrbach, Dennis R. Soil tillage. Ch. 8, Engineering Principles of Agricultural Machines $2^{\text {nd }}$ ed., 169-230 (2006).

18. R.B. Zhelukevich. The destruction of frozen soil with disk cutters; SibADI. Omsk, 22 (1983).

19. R.B. Zhelukevich, Yu.F. Kaiser. Drilling head with disk tool, Political transport systems: materials of the V All-Russian. NTK, at 2 h. Part 2. Krasnoyarsk: Sib. Feder. un-t, 224-228 (2007).

20. B.G. Ziganshin, S.M. Yakhin, R.Kh. Gainutdinov. Theoretical substantiation of the diameter of the ellipsoidal disk of a rotary tool for surface tillage, Bulletin of the Bashkir State Agrarian University 2 (42), 71-74 (2017). 